\title{
A PRODUÇÃO DE MATERIAIS DIDÁTICOS: ENTREVISTA COM NEUS SANS BAULENAS
}

\author{
LA PRODUCCIÓN DE MATERIALES DIDÁCTICOS: ENTREVISTA A NEUS SANS \\ BAULENAS
}
THE PRODUCTION OF TEACHING MATERIALS: AN INTERVIEW WITH NEUS SANS BAULENAS

\author{
Fátima A. T. Cabral BRUNO ${ }^{1}$
}

RESUMO: Esta entrevista com a professora de espanhol como língua estrangeira (E/LE) Neus Sans Baulenas, autora de materiais didáticos de Espanhol como Língua Estrangeira da Editora Difusión da Espanha, tem como objetivo conhecer sua trajetória como escritora há mais de 36 anos e saber o que pensa sobre sua produção editorial. A entrevista também revela a percepção da autora sobre as tendências metodológicas atuais no ensino de línguas e sua opinião sobre questões relacionadas a certas políticas editoriais contemporâneas.

PALAVRAS-CHAVE: Material didático. Produção editorial. Tendências metodológicas. Política editorial.

RESUMEN: Esta entrevista a la profesora de español Neus Sans Baulenas, autora de materiales didácticos de Español como Lengua Extranjera, actualmente de la Editorial Difusión de España, tiene como objetivo conocer su trayectoria como escritora desde hace más de 36 años y saber lo que piensa sobre su producción editorial. La entrevista las actuales tendencias metodológicas y además registrar su opinión sobre cuestiones relativas a determinadas políticas editoriales en la contemporaneidad.

PALABRAS CLAVE: Material didáctico. Producción editorial. Tendencias metodológicas. Política editorial.

ABSTRACT: This interview with the Spanish teacher Neus Sans Baulenas, author of didactic materials of Spanish as a Foreign Language published by Editorial Difusión from Spain, aims at knowing about her career as a writer for more than 36 years and about what she thinks of her editorial production. The interview also reveals the author's perception of the current methodological tendencies in language teaching and her opinion on issues related to certain editorial policies in the present context.

KEYWORDS: Didactic material. Editorial production. Methodological tendencies. Editorial policy.

${ }^{1}$ Universidade de São Paulo - (USP), São Paulo, São Paulo - SP - Brasil. Professora Doutora de Graduação e Pós-Graduação em Língua Espanhola. Departamento de Letras Modernas. ORCID: <http://orcid.org/0000-00022892-6476>.E-mail: fcabralbruno@usp.br 


\section{Introducción}

El 29 de mayo de 2018, echándole un vistazo a la red social Facebook, me deparé con un post de Neus Sans Baulenas, profesora y autora de materiales didácticos de Español como Lengua Extranjera (E/LE), actualmente de la Editorial Difusión, que me llevó a procurarla para una entrevista, en noviembre del mismo año. Dicha entrevista ocurrió durante mi estancia en Barcelona por ocasión de una movilidad académica en la Universitat de Barcelona (UB), una colaboración entre la UB y la Universidade de São Paulo, donde soy docente, de esta última, de las disciplinas de Lengua Española, tanto de Licenciatura como de Postgrado. En el muro de la Prof ${ }^{a}$ Neus se leía y se veía lo siguiente: "Poniendo orden en la "egoteca", con un ejemplar de cada edición. Echo cuentas: iii;36 años escribiendo ele!!!!”

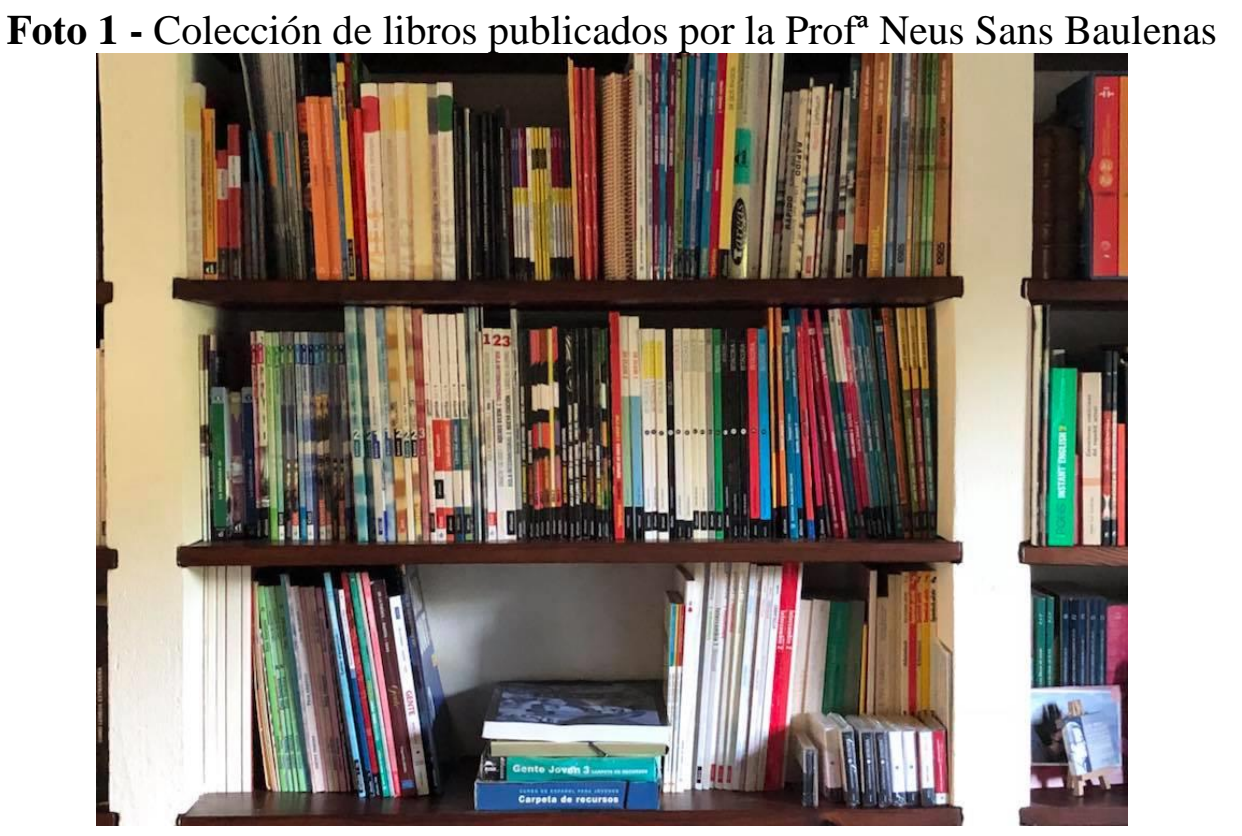

Fuente: Muro del Facebook de la Prof $^{a}$ Neus Sans Baulenas (2018). Disponible: https://www.facebook.com/photo.php?fbid=10214135899565017\&set=a. $1872763812360 \&$ type $=3 \&$ th eater

Me di cuenta, en la época, de que Neus Sans Baulenas lleva tantos años “escribiendo ELE" como llevo yo enseñando español como lengua extranjera en Brasil. Por otro lado, en la secuencia del muro de la Prof ${ }^{a}$ Neus me llamó la atención, entre muchos otros, el comentario del Prof. Jan Peter Nauta sobre los libros que le cambiaron la vida: "Viendo el rosado y el verde de la primera repisa me vienen a la memoria los tiempos en que todo empezó a cambiar... ningún manual me cambió la vida tanto como lo hicieron aquellos tomos.” Como se leerá en la entrevista de la Prof ${ }^{a}$ Neus, materiales como Para empezar (autoría del Equipo Pragma, 
compuesto por Ernesto Martín Peris, Lourdes Miquel López, Terencio Simón Blanco y Marta Topolevsky Bleger, editado por la Editorial Edelsa), Esto funciona (Equipo Pragma, por Edelsa) y ¿A qué no sabes...? (escrito con Lourdes Miquel López y también editado por la Edelsa) causaron, en los años 80 e inicio de los 90, gran impacto en las clases de E/LE, no solo en aquellas tierras en que se enseñaba E/LE, sino también en Brasil, porque proponían no solo un cambio de enfoque, de los que interpreto como Estructuralistas (Traducción, Método Directo etc.) al Comunicativo, sino también que les inspiraban a los docentes a arriesgarse por nuevos caminos pedagógicos. En función del bonito recuerdo del Prof. Nauta, retomo un fragmento mío de Bruno (2018, p. 397) que pone de relieve la importancia de un profesor analíticoreflexivo frente a un libro didáctico:

Lembro-me especificamente do advento da abordagem comunicativa. Naquela época, usei LDs [livros didáticos] espanhóis autodenominados comunicativos e me deparava nas diferentes unidades com determinadas páginas enigmáticas, pois não dava para saber o que teria que fazer com elas. Naquela época, é bom explicar que, na maioria das vezes, não se tinha acesso ao livro do professor. Ao analisar aquelas páginas, eu tinha que pensar em como iria utilizá-las em aulas de modo a atender os objetivos daquela unidade e do curso que eu estava ministrando. Tratava-se de um exercício profissional pessoal em que podia problematizar, ser subjetiva, livre e (re)criadora (BRUNO, 2018, p. 397).

Esta entrevista a Neus Sans, además de llevarnos a un pequeño paseo por la historia de la relación de la autora con su extensa experiencia en la producción de materiales didácticos de E/LE hasta los días de hoy, según se puede observar en la foto de su "egoteca", nos ayuda a reflexionar sobre el dicho y el hecho en las clases de E/LE, ya que la profesora-escritorainvestigadora observa de modo muy realístico el fallo que existe todavía entre los principios metodológicos suscritos por los profesores y sus respectivas prácticas docentes, muchas veces ancladas en creencias mecanicistas, repetitivas sobre la enseñanza-aprendizaje de E/LE. Por otro lado, estoy totalmente de acuerdo con la Prof ${ }^{a}$ Neus cuando se refiere a las nuevas herramientas tecnológicas:

Sabemos muy poco todavía sobre cómo se aprende una lengua extranjera y frecuentemente nos olvidamos de esos principios consensuados. La mitificación de las herramientas tecnológicas, por ejemplo, nos devuelve, a veces, a prácticas y concepciones de la lengua totalmente obsoletas.

En vista de todo esto y de otros aportes tan importantes como, por ejemplo, sobre la relación autor-editorial, les invito a leer la entrevista de Neus Sans Baulenas que ha trabajado intensamente con diferentes coautores ( es bueno registrarlo!) y equipo editorial para 
brindarnos con materiales didácticos que intentan reflejar siempre una confluencia entre teoría y práctica.

\section{LA ENTREVISTA}

Fátima A. T. Cabral Bruno: ¿Cómo, cuándo y por qué empezaste en el área editorial? Neus Sans Baulenas: A finales de los años 70, y principios de los 80, un grupo de jóvenes profesores inquietos de Español para extranjeros, convivíamos en la Escuela Oficial de Idiomas de Barcelona con profesores de otras lenguas (en particular de inglés, de francés y de alemán). Para la enseñanza de esas lenguas se disponía ya de material abundante e innovador. El panorama del ELE, en cambio, era un auténtico páramo. Casi por la necesidad inmediata de atender nuestras clases, nos pusimos a diseñar materiales. No teníamos ni la menor idea del impacto que iban a tener en la profesión nuestras primeras publicaciones: A que no sabes?, Para empezar, Esto funciona ...

Empezamos a trasladar al ELE lo que ya se estaba haciendo y publicando para las otras lenguas. En esencia era algo muy concreto: empezar a entender las lenguas vivas como herramientas de comunicación. Y como tales debían describirse y enseñarse. Recordemos que en esa época las primeras directrices del Consejo de Europa, a través del Proyecto de Lenguas Vivas y los Niveles Umbral, ya están muy definidas. Los primeros enfoques comunicativos son imparables, por mucha resistencia que inspiraran entre algunos docentes.

Fátima A. T. Cabral Bruno: ¿Cuántos y qué tipo de libros publicaste? ¿Por qué?

Neus Sans Baulenas: He perdido la cuenta... Pero creo que he logrado publicar solo cuando tenía la sensación de que había cosas nuevas que plantear. Cada método, cada manual ha supuesto un nuevo reto. Me gustan los retos.

Fátima A. T. Cabral Bruno: A lo largo de los años, ¿qué cambios fueron más significativos en los materiales didácticos en general y en los tuyos? ¿Por qué?

Neus Sans Baulenas: Desde esa primera época, un momento de inflexión importante, a mi modo de ver, es, a mediados de los 90, cuando logramos entender que la comunicación no solo es el objetivo de la enseñanza-aprendizaje sino también el camino inevitable para el aprendizaje. Es decir, no solo aprendemos lenguas para usarlas en relaciones de interacción social sino que solamente a través de la interacción aprendemos. Eso lo cambia todo. Las 
actividades de aula ya no pueden limitarse a reproducir la comunicación, a ser un teatrillo donde se imitan situaciones de comunicación- sino que deben provocar interacciones significativas y relevantes en el grupo clase. Las buenas tareas son aquellas que aportan un gran caudal de lengua meta porque provocan la comunicación en el aula. El alumno siente que tiene algo que decir y que quiere compartirlo.

Fátima A. T. Cabral Bruno: ¿Cómo caracterizarías las tendencias metodológicas o abordajes de los materiales didácticos actuales? ¿Por qué?

Neus Sans Baulenas: Seguramente sigue habiendo una brecha importante entre los principios metodológicos que la mayor parte de profesores teóricamente suscribe y la realidad de las prácticas docentes, muy ancladas en creencias mecanicistas sobre el aprendizaje, en aulas gestionadas todavía muy frontalmente, donde la corrección morfosintáctica es el eje indiscutido. Falta mucho por hacer. El sunami tecnológico nos da nuevas herramientas y nuevas situaciones de aprendizaje. $Y$, sin duda, hay una infinidad de experiencias interesantes: se gamifican clases, se usa tecnología, se intenta articular la presencialidad con la autonomía del aprendiz con las clases invertidas, etc. pero, en el fondo, queda mucho por hacer. Sabemos muy poco todavía sobre cómo se aprende una lengua extranjera y frecuentemente nos olvidamos de esos principios consensuados. La mitificación de las herramientas tecnológicas, por ejemplo, nos devuelve, a veces, a prácticas y concepciones de la lengua totalmente obsoletas.

Fátima A. T. Cabral Bruno: ¿Los materiales didácticos impresos, como producto de editoriales, van a acabar a causa de internet y de los recursos educacionales abiertos (REA)? ¿Por qué?

Neus Sans Baulenas: Es un proceso mucho más lento de lo que se había imaginado desde el sector editorial. El papel sigue siendo reclamado por alumnos y profesores. El manual ofrece un orden en la selección de objetivos, una "consultabilidad" que reclaman unos y otros, frente al inasible alubión de materiales a los que lanza el Internet. Sin duda vamos a fórmulas mixtas.

Fátima A. T. Cabral Bruno: ¿Hasta qué punto el autor de material didáctico es libre para escribir; esto es, no necesita seguir las olas metodológicas, muchas veces, impuestas por las editoriales?

Neus Sans Baulenas: Siempre existe la tensión entre lo que "piden los mercados" (más de lo mismo) y la innovación, con todos los riesgos que entraña. En el mundo del ELE, sin embargo, 
yo diría que los autores hemos sido bastante libres a la hora de proponer alternativas metodológicas. Recordemos, por ejemplo, la llegada de los enfoques mediante tareas, que ha conseguido en pocos años revolucionar las aulas. Los profesores acaban adoptando aquello que hace mejorar sus clases. Y los materiales que activan la creatividad y la implicación del alumno sin duda las mejoran. Por eso hay que experimentar, innovar, probar cosas nuevas... $Y$ las editoriales especializadas en ELE lo han permitido sin ser esclavas de modas con fecha de caducidad.

Fátima A. T. Cabral Bruno: ¿Qué trabajo has desarrollado con relación a la formación de nuevos autores de materiales didácticos?

Neus Sans Baulenas: El diseño de materiales es inevitablemente un trabajo de equipo. Siempre hemos ido incorporando a los equipos autores jóvenes y juntos hemos ido creciendo. Es en la discusión sobre cada página de un manuscrito, de cada actividad, de cada ejemplo es donde se forma un autor. Diseñar materiales es una compleja alquimia entre teoría y artesanía.

Fátima A. T. Cabral Bruno: ¿La relación entre el equipo editorial y el autor ha cambiado a lo largo de los años? ¿Cómo y por qué?

Neus Sans Baulenas: Obviamente, hay contextos muy diversos. Solo puedo hablar de mi caso. Tenemos más medios técnicos pero también más presión. Hay mucha oferta, mucha competencia, y a veces se añoran los tiempos en que el autor era más valiente. Se publica mucho pero muchos materiales son totalmente irrelevantes, clones innecesarios.

Fátima A. T. Cabral Bruno: ¿Qué estás produciendo actualmente? ¿Por qué?

Neus Sans Baulenas: En la actualidad estoy trabajando mucho en la creación de materiales audiovisuales. Vivimos en un mundo audiovisual y la educación no puede permanecer ajena a ello. En el caso de las lenguas extranjeras es evidente que los materiales audiovisuales tienen muchas ventajas. Y hay poco material audiovisual de calidad, que enganche a un aprendiz con un listón audiovisual muy alto.

$Y$ en el ámbito de los manuales, en nuestro último curso, Bitácora, nos hemos adentrado en una nueva priorización del componente léxico, que ha sido la cenicienta del ELE, sin dejar de profundizar en aspectos esenciales como la dimensión discursiva de las lenguas, el desarrollo de la autonomía del aprendiz y la interacción significativa como clave de los procesos de enseñanza-aprendizaje. 
Fátima A. T. Cabral Bruno: De todo lo que publicaste, ¿cuál consideras el mejor material y por qué?

Neus Sans Baulenas: Uno siempre tiene más cariño a sus últimos proyectos. Bitácora es para mí el más maduro, el que mejor responde a todas las preguntas que nos hemos hecho en las últimas décadas. Pero soy muy autocrítica con todo desde el día que un material sale de la imprenta. Como dije antes, queda mucho por hacer, por investigar, por experimentar. Todo es infinitamente mejorable.

Foto 2 - Profesoras Fátima A. T. Cabral Bruno y Neus Sans Baulenas (de izquierda a derecha). En la editorial Difusión, Barcelona/España.

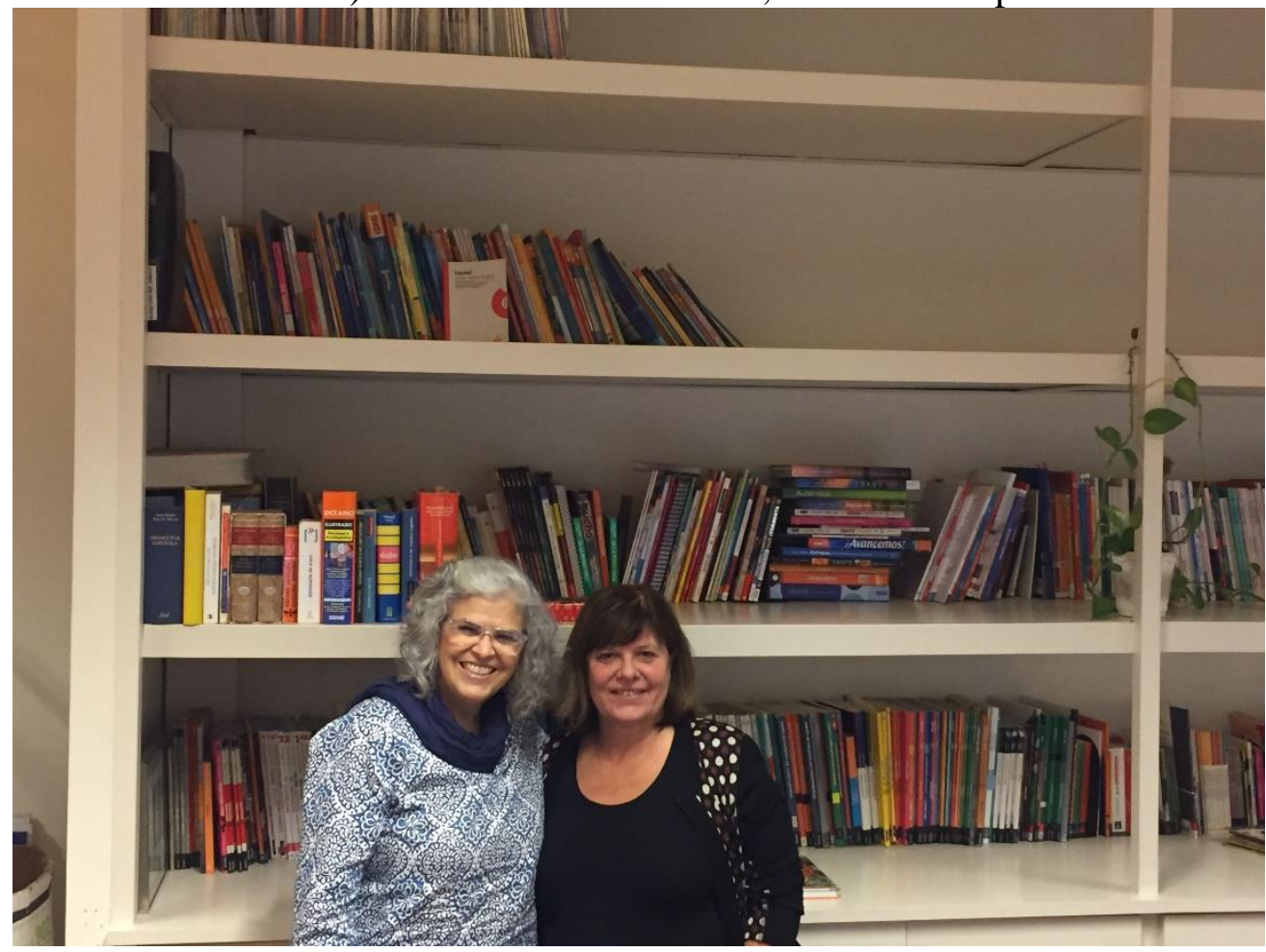

Fuente: Archivo personal (2018)

\section{Para terminar}

Para finalizar este artículo-entrevista, me parece importante poner de relieve que un libro didáctico y, por extensión cualquier recurso didáctico:

também é importante por seu aspecto político e cultural, na medida em que reproduz e representa os valores da sociedade em relação à sua visão da ciência, da história, da interpretação dos fatos e do próprio processo de transmissão do conhecimento. (OLIVEIRA; GUIMARÃES; BOMÉNY, 1984, p. 11) 
Y que, por eso, como nos apunta la Prof ${ }^{a}$ Neus, es o debería ser "infinitamente mejorable". En ese sentido, el autor de materiales didáctico debería ser "más valiente", retomando las palabras de Neus, ya que como ella misma nos declara "Se publica mucho pero muchos materiales son totalmente irrelevantes, clones innecesarios." Lo que me lleva a preguntarme la razón de la falta de valentía. En Brasil, en el caso de E/LE, parece ser por dos motivos, como lo subrayo en Bruno (2018), por un lado, por los modismos metodológicos y, por otro, por la presión de las editoriales motivadas por el Plano Nacional do Livro Didático (PNLD 2018, 2015) y que, en su mayoría, no son especializadas en la enseñanza/aprendizaje de E/LE.

\section{REFERÊNCIAS}

BRUNO, Fátima A. T. Cabral. O livro e a reforma do pensamento. In: BARROS, Cristiano Silva de; COSTA, Elzimar Goettenauer de Marins; FREITAS, Luciana Maria Almeida de. (Orgs.). O livro didático de espanhol na escola brasileira. Campinas: Pontes Editores, p. 387-404, 2018.

BRASIL. Ministério da Educação. Fundação Nacional de Desenvolvimento da Educação. Edital de convocação para o processo de inscrição e avaliação de obras didáticas para o Programa Nacional do Livro Didático - PNLD 2018. Edital de convocação 04/2015 CGPLI. Brasília, 2015. Disponível em: http://222.fnde.gov.br/programas/livro-didatico/livrodidatico-editais. Acesso em: 24 set. 2016.

OLIVEIRA, João Batista Araújo e; GUIMARÃES, Sônia Dantas; BOMÉNY, Helena Maria Bousquet. A política do livro didático. São Paulo: Summus; Campinas: Ed. da Universidade de Campinas. 1984.

\section{Como citar este artigo:}

BRUNO, Fátima A. T. Cabral. A produção de materiais didáticos: entrevista com Neus Sans Baulenas. Rev. EntreLínguas, Araraquara, v. 5, n. 1, p. 92-99, jan./jun. 2019. E-ISSN: 24473529. DOI: 10.29051/el.v5i1.12410

Submetido em: 03/01/2019

Aprovado em: 10/03/2019

Publicado em: 30/04/2019 